\title{
MicroRNA-133a and microRNA-326 co-contribute to hepatocellular carcinoma 5-fluorouracil and cisplatin sensitivity by directly targeting B-cell lymphoma-extra large
}

\author{
JIN MA ${ }^{1}$, TING WANG ${ }^{1}$, RUI GUO ${ }^{1}$, XIAOYAN YANG $^{1}$, JIE YIN $^{1}$, JIA YU $^{1}$, QIONG XIANG ${ }^{1}$, \\ XIA PAN $^{1}$, XUYU ZU ${ }^{2}$, CUIYING PENG ${ }^{1}$, HUIFANG TANG ${ }^{3}$ and XIAOYONG LEI ${ }^{1}$ \\ ${ }^{1}$ Institute of Pharmacy and Pharmacology, University of South China; ${ }^{2}$ Institute of Clinical Medicine; \\ ${ }^{3}$ Cardiology Department, The First Affiliated Hospital of University of South China, Hengyang, Hunan 421001, P.R. China
}

Received June 16, 2014; Accepted July 10, 2015

DOI: $10.3892 / \mathrm{mmr} .2015 .4134$

\begin{abstract}
Chemotherapy is one of the most common treatments used for hepatocellular carcinoma (HCC), which effectively improves outcome and reduces tumor recurrence. However, the drug resistance mechanisms involved in chemotherapy, which is the predominant challenge in HCC treatment, remain to be fully elucidated. Therefore, there is an urgent requirement for the identification of novel therapeutic strategies or drugs. MicroRNAs (miRs) have become an area of interest, and in the present study, the effects of miR-133a and miR-326 on HepG2 cells, and their function on B-cell lymphoma-extra large (Bcl-xl) in HepG2 cells were investigated. Using computational programs, $\mathrm{Bcl}-\mathrm{xl}$ was predicted as the common target gene of miR-133a and miR-326. A dual-luciferase reporter assay was used to verify the target genes of miRs. The mRNA and protein levels of Bcl-xl were observed to be downregulated following transfection with miR-133a or miR-326 mimics. Combining miR-133a or miR-326 with 5-fluorouracil (5-FU) or cisplatin (DDP) resulted in increased cell death. The results of the present study indicated that miR-133a, miR-326 and Bcl-xl acted protectively against the apoptosis, induced by 5-FU or DDP, in HepG2 cells. This suggested the potential use of miRs either as ancillary anti-cancer drugs or as anti-cancer drugs themselves.
\end{abstract}

\section{Introduction}

Apoptosis is a conserved and irreversible process, which allows cells to undergo a tightly controlled form of death and is essential for normal development and homeostasis $(1,2)$. Apoptosis

Correspondence to: Mr. Xiaoyong Lei, Institute of Pharmacy and Pharmacology, University of South China, 28 Changsheng Road, Hengyang, Hunan 421001, P.R. China

E-mail: leixiaoyong@aliyun.com

Key words: microRNA, hepatocellular carcinoma, 5-fluorouracil, B-cell lymphoma-extra large, cisplatin is a process, which involves the body removing unused or harmful cells in order to protect itself, and the deregulation of apoptosis is the predominant obstacle in the successful treatment of cancer. Inhibiting the expression of anti-apoptotic factors or inducing pro-apoptotic factors contributes to the apoptosis of cancer cells (3). The B-cell lymphoma-2 (Bcl-2) apoptotic protein family, one of the key apoptotic gene families, contains pro-apoptotic and anti-apoptotic members, all of which act as critical regulators of apoptosis $(4,5)$. Members of the $\mathrm{Bcl}-2$ protein family include three subgroups of proteins, which either promote cell survival, including Bcl-2 and B-cell lymphoma-extra large (Bcl-xl), initiate cell killing, including Bcl-2-like protein 11 and $\mathrm{BH} 3$ interacting-domain death agonist or activate the effector pathways of apoptosis, including $\mathrm{B}$-cell-associated $\mathrm{X}$ protein (Bax) and $\mathrm{Bcl}-2$ homologous antagonist/killer (6).

Previous studies have demonstrated that small non-protein-coding molecules, termed microRNAs (miR) serve key roles in homeostatic processes. These processes include cell development, proliferation and apoptosis (7-9), in addition to the regulation of gene expression at the translational level (10) by recognizing and binding to the $3^{\prime}$ untranslated region (3'UTR) of the target mRNA with its seed region (11). However, the role of miR in apoptosis remains to be fully elucidated. Previous studies have demonstrated that miRs are important in this process, particularly in regulating the Bcl-2 gene family (12-14). miR-29 has been reported to promote apoptosis through a mitochondrial pathway, which involves myeloid leukemia cell (Mcl)-1 and Bcl-2 (15), while the downregulation of miR-125b, has been reported to inhibit the expression of Bcl-2 and promote apoptosis in hepatocellular carcinoma (HCC) (16). It has been reported that miR-125b is able to reduce the cellular proliferation and cell cycle progression of HCC cells by targeting Mcl-1 and the interleukin 6 receptor (17). Bcl-w downregulation by miR-122 has been reported to result in apoptosis in the Hep3B and HepG2 HCC cell lines (18). miRs are also able to modulate cancer cell sensitivity to anticancer therapy via the Bcl-2 gene family (19). Qiu et al (20) reported that miR-503 regulates cell apoptosis by targeting $\mathrm{Bcl}-2$, and modulates the resistance of non-small cell lung cancer cells to cisplatin (DDP). miRNA-195 is 
downregulated in BEL-7402/5-fluorouracil (5-FU) cells, and overexpression of miRNA-195 sensitizes BEL-7402/5-FU cells to 5-FU by targeting Bcl-w to increase cell apoptosis (21).

$\mathrm{Bcl}-\mathrm{xl}$, an anti-apoptotic Bcl-2 family member, is regulated by miRs $(22,23)$. However, the mechanisms underlying the association between miRNAs and Bcl-xl remain to be elucidated. The present study aimed to investigate whether miR-133a and miR-326 contribute to the regulation of chemotherapy resistance, mediated by $\mathrm{Bcl}-\mathrm{xl}$, by examining cell apoptosis in the HepG2 HCC cell line.

\section{Materials and methods}

Materials. The human HepG2 HCC cell line was purchased from the Cell Bank of the Chinese Academy of Sciences (Shanghai, China). Dulbecco's modified Eagle's medium (DMEM) and fetal bovine serum (FBS) were obtained from GE Healthcare Life Sciences (Beijing, China). 5-FU and DDP were purchased from Sigma-Aldrich (St. Louis, MO, USA). The miR mimics were purchased from Shanghai GenePharma Co., Ltd. (Shanghai, China).

Identification of miR target sites. The miRanda (http://www.microrna.org/), TargetScan (http://www. targetscan.org/vert_61/), Pictar (http://pictar.mdc-berlin. de/cgi-bin/PicTar_vertebrate.cgi) and miRBase (http://www. mirbase.org/search.shtml) algorithms were used to predict the putative target genes of miR-133a and miR-326.

Cell culture. The human HepG2 HCC cell line was maintained in DMEM, supplemented with $4.5 \mathrm{~g} / 1$ glucose, $10 \% \mathrm{FBS}$ and $1 \%$ penicillin/streptomycin (GE Healthcare Life Sciences) in a humidified atmosphere containing $5 \% \mathrm{CO}_{2}$ at $37^{\circ} \mathrm{C}$.

Construction of 3'UTR-luciferase reporter plasmids. The full-length Bcl-xl cDNA construct containing the entire 3'UTR was synthesized and cloned into a pcDNA3.1 plasmid (Landbiology, Guangzhou, China). Site directed mutagenesis using the QuikChange Site-Directed Mutagenesis kit (Agilent Technologies GmbH, Waldbronn, Germany) using the following primers (Shanghai Novland Co., Ltd., Shanghai, China): Forward, 5'-GCTCCCATGACCATACTGAGCCTG GTTCTGGGCCCAAGACAGATGCC-3' and reverse 5'-GG CATCTGTTCTTGGGCCCAGAACCAGGCTCAGTATG GTCATGGGAGC-3'. The 3'UTR (wild-type and mutant) of Bcl-xl was synthesized and was inserted downstream of the Renilla luciferase gene of the dual-luciferase. The primer for Bclxl was designed, and targeted fragments were obtained by PCR amplification (Stratagene, Santa Clara, CA, USA). Subsequently, the specificity of the PCR primers was analyzed through digesting PCR products with restriction endonuclease. The target gene was then linked with the vector and recombinant plasmids were amplified by transformation into $E$. coli (Landbiology). Positive clones were selected randomly and sequenced for verification.

Luciferase assay. The wild-type and mutant $\mathrm{Bcl}-\mathrm{xl}$ 3'UTR-luciferase reporter plasmids were co-transfected with a miR-133a or miR-326 mimic (Shanghai GenePharma, Co., Ltd.) into the HEK923 cells (American Type Culture
Collection, Manassas, VA, USA) using Lipofectamine 2000 (Invitrogen Life Technologies, Carlsbad, CA, USA). At 48 h post-transfection, the cells were assayed for luciferase activity using a Dual-Luciferase Assay system (Promega Corporation, Madison, WI, USA), according to the manufacturer's instructions. The Renilla luciferase activities were normalized to the corresponding activities of firefly luciferase. For each transfection, the average luciferase activity was calculated from three replicates.

Reverse transcription-quantitative polymerase chain reaction (RT-qPCR) analysis. RT-qPCR was performed, as previously reported (24). Total RNA was extracted from the cells using TRIzol reagent (Tiangen Biotech Co., Ltd., Beijing, China), according to the manufacturer's instructions. The primer sequences for Bcl-xl, Bax, GAPDH, miR-133a, miR-326 and U6 were as follows: Bcl-xl, forward 5'-GCT GGTGGTTGACTTTCTCTCCTAC-3' and reverse 5'-CCT CAGTCCTGTTCTCTTCCACATC-3'; Bax, forward 5'-CCC TTTTCTACTTTGCCAGCA-3' and reverse 5'-GGAGTC TCACCCAACCACCC-3'; GAPDH, forward 5'-CATGAG AAGTATGACAACAGCCT-3' and reverse 5'-AGTCCTTCC ACGATACCAAAGT-3'; miR-133a, forward 5'-TCATAT TTGGTCCCCTTCAACC-3' and reverse 5'-TATCGTTGT TCTCCACTCCTTCAC-3'; miR-326, forward 5'-ACTGTC CTTCCCTCTGGGC-3' and reverse 5'-AATGGTTGTTCT CCACTCTCTCTC-3'; and U6 small nuclear RNA, forward 5'-ATTGGAACGATACAGAGAAGATT-3' and reverse 5'-GGAACGCTTCACGAATTTG-3'. The U6 small nuclear RNA was used as an internal control for miR quantification. To determine the mRNA expression levels of Bcl-xl and Bax, the total RNA were reverse transcribed into cDNA using a Custom Reverse Transcription kit. The qPCR analyses of Bcl-xl and Bax were performed using the above-mentioned primers, and normalized to GADPH. SYBR green qPCR was performed on a MX3000P PCR System (Agilent Technologies, Inc., Santa Clara, CA, USA). The cycling programme consisted of 40 cycles, with each cycle consisting of $3 \mathrm{~min}$ at $95^{\circ} \mathrm{C}, 12 \mathrm{sec}$ at $95^{\circ} \mathrm{C}$ and $40 \mathrm{sec}$ at $62^{\circ} \mathrm{C}$. The relative expression ratios of Bcl-xl, Bax, miR-133a, miR-326 were calculated using the $2^{-\Delta \Delta C t}$ method (25).

Cell transfection. The cells were transfected using Lipofectamine 2000 transfection reagent according to the manufacturer's instructions (Invitrogen Life Technologies) The sequences for the two miRNA mimics were as follows: miR-133a: 5'-uuugguccccuucaaccagcug-3'; miR-326: 5'-ccucug ggcccuuccuccag-3'. At 1 day prior to transfection, the cells were plated in the appropriate quantity of growth medium without antibiotics in order to reach $80-90 \%$ confluency at the time of transfection. For each transfection sample, miRNA mimicsLipofectamine 2000 complexes were prepared as follows: miRNA mimics (Shanghai GenePharma, Co., Ltd.) were diluted in the appropriate quantity of Opti-minimum essential medium (MEM; Gibco-BRL, Grand Island, NY, USA) without serum and mixed gently. Lipofectamine 2000 was diluted in the appropriate quantity of Opti-MEM without serum then mixed gently. The samples were incubated for $5 \mathrm{~min}$ at room temperature. The diluted miRNA mimics were then combined with the diluted Lipofectamine 2000 and incubated for $20 \mathrm{~min}$ 


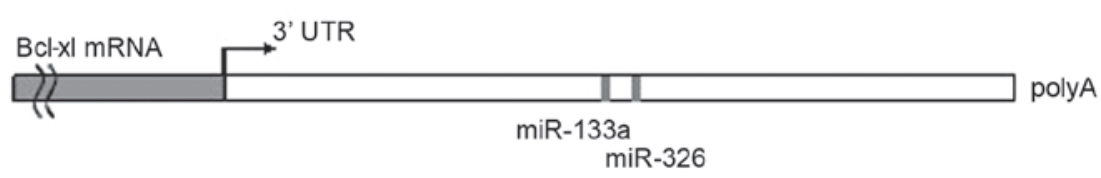

\section{Conserved}

Position 765-771 of BCL2L1 3' UTR

hsa-miR-133a

Conserved

Position 791-797 of BCL2L1 3' UTR

hsa-miR-326

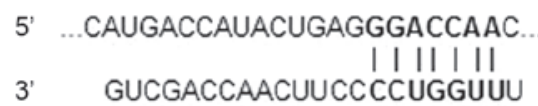

3' GUCGACCAACUUCCCCUGGUUU

Figure 1. Putative miR-133a and miR-326 targeted sequence in the Bcl-xl gene. TargetScan was used to predict a common target gene for miR-133a and miR-326. miR, microRNA; UTR, untranslated region.

at room temperature to allow complex formation to occur. The miRNA mimics-Lipofectamine 2000 complexes were added to each well containing cells and medium. The cells were incubated at $37^{\circ} \mathrm{C}$ in a $\mathrm{CO}_{2}$ incubator until harvesting of cells and the assay for the target gene.

Western blot analysis. At $48 \mathrm{~h}$ post-transfection, the cell lysates were prepared by lysis in radioimmunoprecipitation assay buffer (CWBIO, Beijing, China) with protease inhibitors. Centrifugation was then performed at 10,000-14,000 x g for $15 \mathrm{~min}$ at $4^{\circ} \mathrm{C}$, and the protein concentration was determined using a bicinchoninic acid assay (CWBIO). The proteins (30-50 $\mu \mathrm{g}$ ) were separated on 10\% SDS-polyacrylamide gels (CWBIO) and were transferred onto polyvinylidene difluoride membranes (EMD Millipore, Billerica, MA, USA). A PageRuler prestained protein ladder (CWBIO) was used as a molecular marker. A single membrane was cut into three parts at the $43 \mathrm{kD}, 30 \mathrm{kD}$ and $20 \mathrm{kD}$ bands, and incubated with anti- $\beta$-actin (1:1,000; cat. no. 12620), anti-Bcl-xl (1:1,000; cat. no. 2764) and anti-Bax (1:1,000; cat. no. 5023) monoclonal rabbit primary antibodies (Cell Signaling Technology, Inc., Danvers, MA, USA), respectively. The proteins were detected using a horseradish peroxidase-conjugated goat anti-rabbit IgG secondary antibody (1:4,000; cat. no. CW0103; CWBIO) and a ChemiLucent ECL Detection system (EMD Millipore, Billerica, MA, USA). Quantification of the protein bands was performed using AlphaImager 2200 software (National Institutes of Health, Bethesda, MD, USA).

MTT analysis. The HepG2 cells in the exponential growth phase were seeded into 96 -well plates at a density of $4 \times 10^{3}$ cells/well and were incubated for $12 \mathrm{~h}$ at $37^{\circ} \mathrm{C}$. The cells were transfected with either the miR-133a mimic, miR-326 mimic or the negative control (NC). At $24 \mathrm{~h}$ post-transfection, various concentrations of 5-FU $(0,5,50$, 500 and $5,000 \mu \mathrm{M})$, or DDP $(0,6.25,12.5,25,50$ and $100 \mu \mathrm{M})$ were added. Subsequent to incubation for $24 \mathrm{~h}, 20 \mu \mathrm{l}$ MTT (Amresco LLC, Solon, OH, USA) solution, at a concentration of $5 \mathrm{mg} / \mathrm{ml}$, was added to each well for $4 \mathrm{~h}$ at $37^{\circ} \mathrm{C}$. The culture medium was then removed, and the insoluble
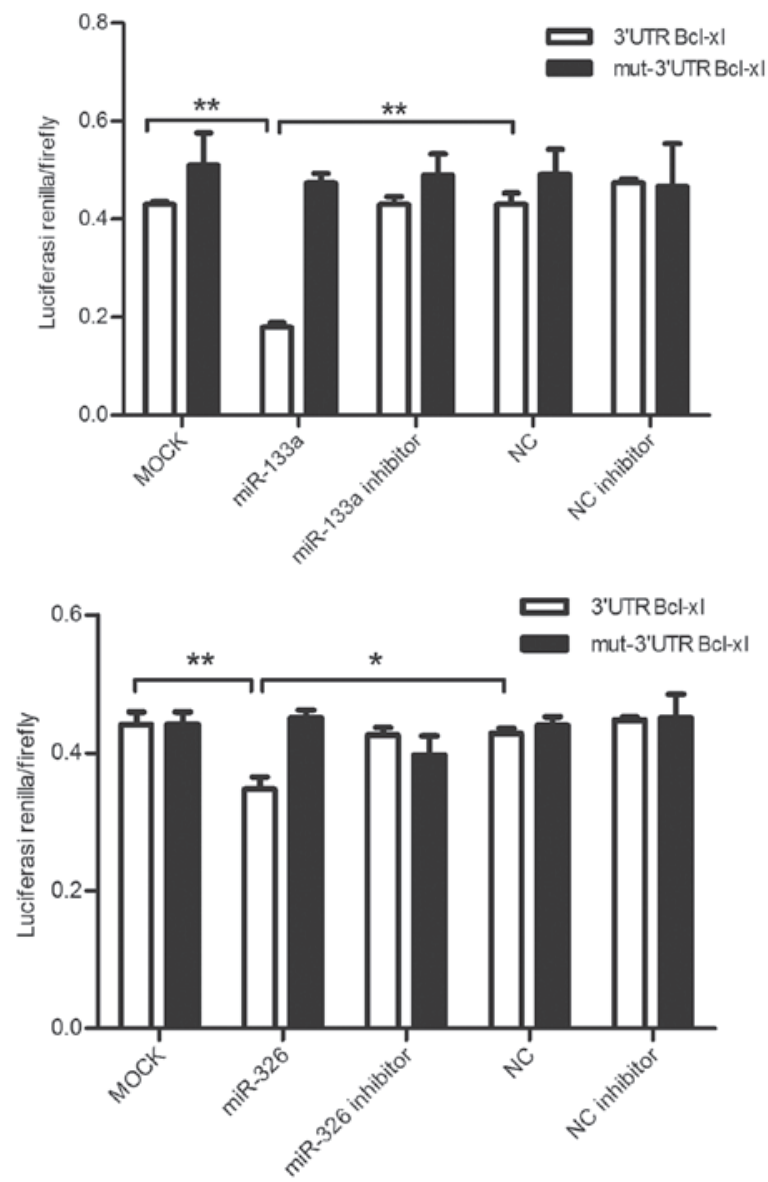

Figure 2. Activities of miR-133a and miR-326 on the 3'UTR of Bcl-xl were assessed using dual-luciferase reporter assays. The Bcl-xl 3'UTR was incorporated into the firefly luciferase gene and run off a single promoter. The constitutively expressed Renilla gene served to normalize the transfections. All the constructs were introduced into HEK293 cells with miR-133a, miR-326 or the negative control, and luminescence was measured after $48 \mathrm{~h}$. miR-133a and miR-326 were found to reduce luciferase levels. The sequence specificity of miR-133a and miR-326 of the Bcl-xl 3'UTR interaction were probed by mutating the seed sequence of miR-133a or miR-326. Compared with the wild-type, the mutated version had a negligible effect on the Bcl-xl 3'UTR, as quantified by levels of luciferase reduction subsequent to transfection. Data are presented as the mean fold reduction \pm standard deviation. All experiments were performed in triplicate $\left({ }^{*} \mathrm{P}<0.05\right.$ and $\left.{ }^{* *} \mathrm{P}<0.01\right)$. miR, microRNA; UTR untranslated region; $\mathrm{NC}$, negative control. 


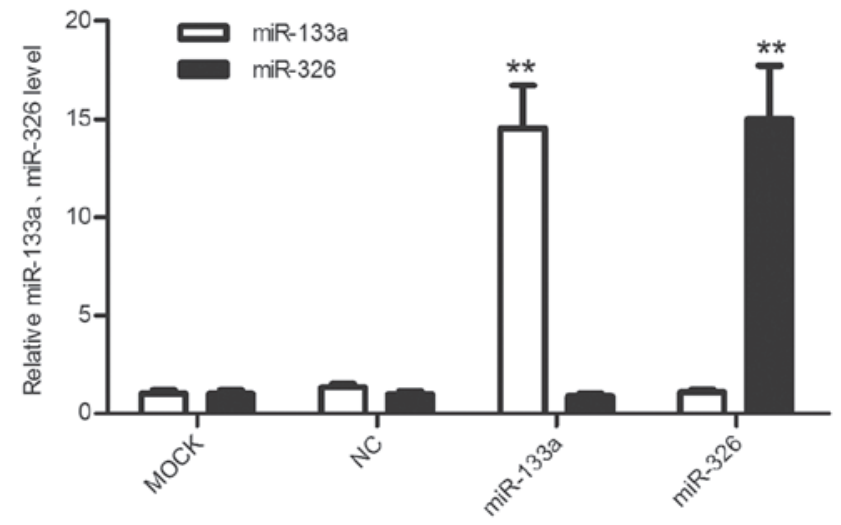

Figure 3. Quantitative expression levels of miR-133a and miR-326 in the mimic-transfected HepG 2 cells was determined by reverse transcription-quantitative polymerase chain reaction. The expression levels were upregulated compared with the $\mathrm{NC}$ and mock cells $\left({ }^{*} \mathrm{P}<0.05\right.$ and ${ }^{* *} \mathrm{P}<0.01$, compared with mock and NC cells). miR, microRNA; NC, negative control; mock, untreated.

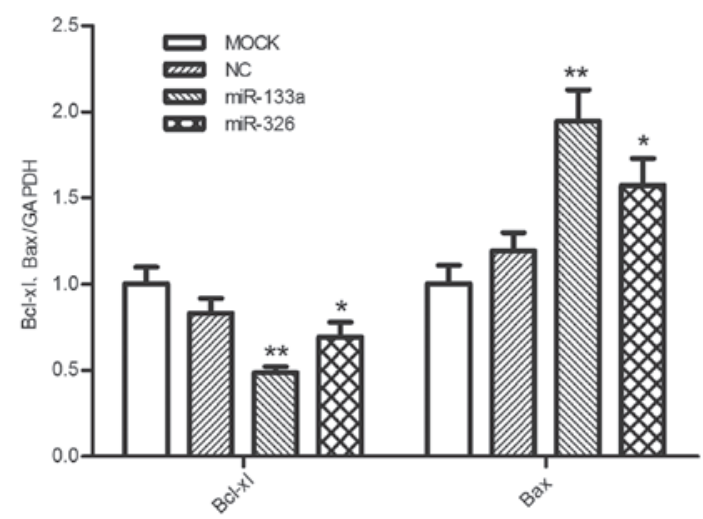

Figure 4. mRNA expression levels of Bcl-xl in the miR-133a and miR-326 mimic-transfected HepG2 cells were determined by reverse transcription-quantitative polymerase chain reaction. The expression levels were downregulated compared with the $\mathrm{NC}$ and mock cells. The opposite results were observed in the mRNA expression levels of $\mathrm{Bax}$ ( $\mathrm{P}<0.05$ and ${ }^{* *} \mathrm{P}<0.01$, compared with mock and NC cells). Data are expressed as the mean \pm standard deviation. miR, microRNA; NC, negative control, mock, untreated.

formazan crystals were dissolved in $150 \mu \mathrm{l}$ dimethyl sulfoxide (Beijing Dingguo Changsheng Biotechnology Co., Ltd., Beijing, China). Following agitation for $10 \mathrm{~min}$, the absorbance at $490 \mathrm{~nm}$ (A490) was optically monitored using a 318-microplate reader (Shanghai Sanco Instrument Co., Ltd., Shanghai, China).

Statistical analysis. SPSS software, version 18.0 (SPSS, Inc., Chicago, IL, USA) was used for statistical analysis. All values are expressed as the mean \pm standard deviation. One-way analysis of variance was performed to evaluate the significance of the differences between samples. $\mathrm{P}<0.05$ was considered to indicate a statistically significant difference.

\section{Results}

miR-133a and miR-326 share a common target gene, Bcl-xl. To examine the mechanism of miR-133a and miR-326, the TargetScan, Pictar, miRanda and miRBase computational programs were used to predict putative terget genes for the
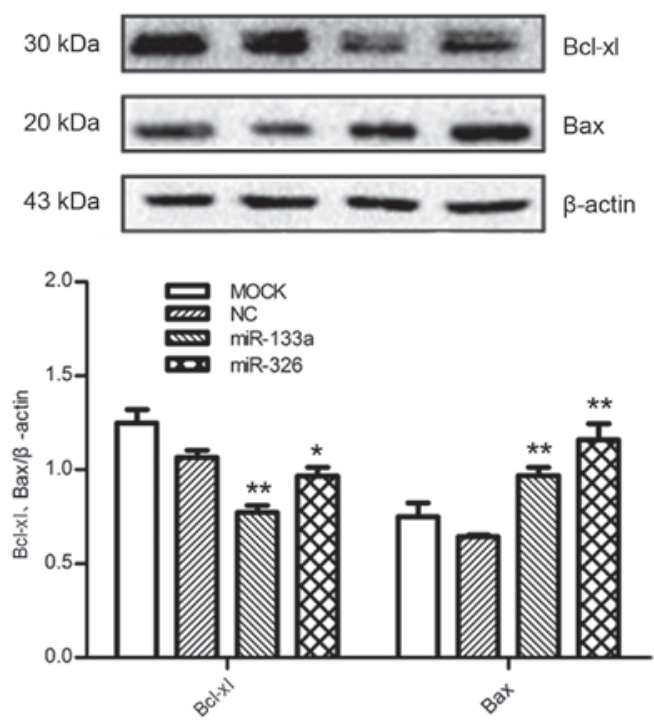

Figure 5. Protein expression levels of Bcl-xl in the miR-133a and miR-326 mimic-transfected HepG2 cells were determined by western blot analysis. The expression levels were downregulated compared with the control cells and untreated cells. The levels of the proteins were quantified using Image software $\left({ }^{*} \mathrm{P}<0.05\right.$ and ${ }^{* *} \mathrm{P}<0.01$, compared with mock and $\mathrm{NC}$ cells). Data are expressed as the mean \pm standard deviation. miR, microRNA; NC, negative control; mock, untreated.
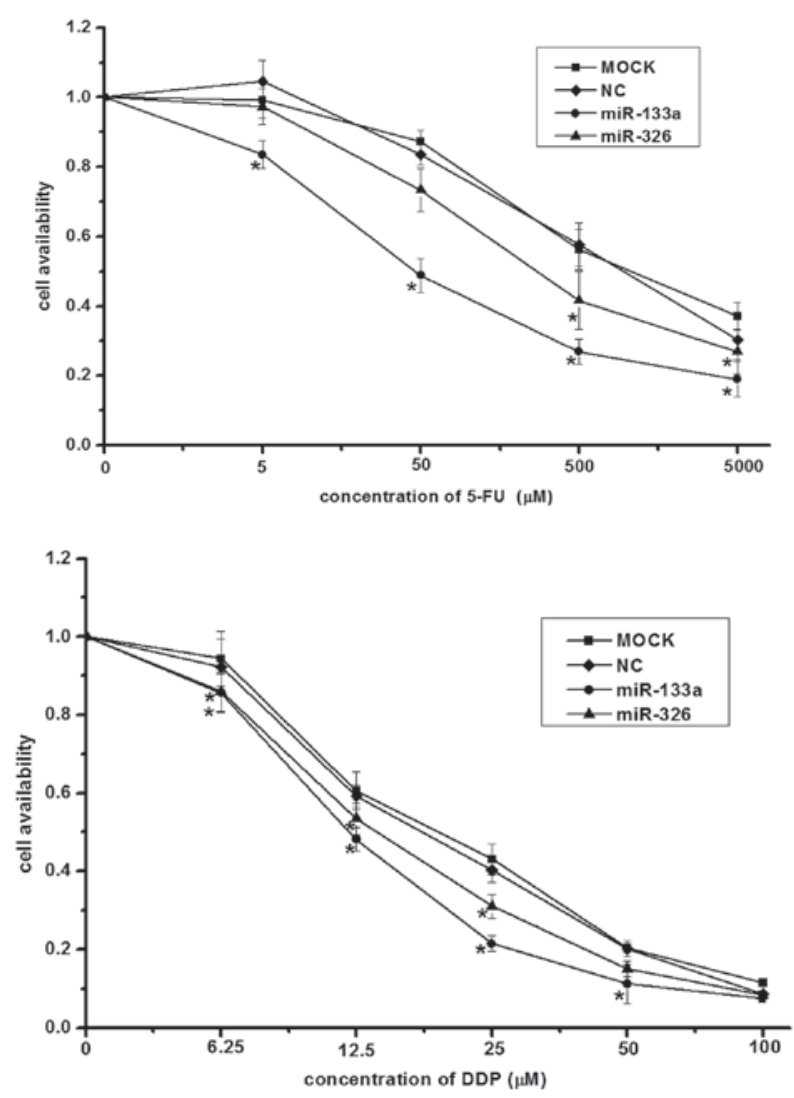

Figure 6. Effects of miR-133a and miR-326 on the sensitivities of HepG2 cells to 5-FU and DDP. The drug resistance of the miR-133a- and miR-326-transfected HepG2 cells, NC-transfected HepG2 cells and untreated HepG2 cells were assessed with increasing concentrations of 5-FU or DDP and measuring the cell survival using an MTT cell proliferation assay $48 \mathrm{~h}$ after treatment. miR-133a and miR-326 in the mimic-transfected HepG2 cells sensitized the cells to 5-FU and DDP ( $\mathrm{P}<0.05$, compared with mock and NC cells). Data are expressed as the mean \pm standard deviation. miR, microRNA; 5-FU, 5-fluorouracil; DDP, cisplatin; NC, negative control; mock, untreated. 
two miRs. The programs predicted the common target gene for miR-133a and miR-326, as the human Bcl-xl gene (Fig. 1).

Confirmation of putative miR binding sites for $m i R-133 a$ and miR-326 using luciferase reporter assays. The present study confirmed the predicted binding sites recognized by miR-133a and miR-326 using the pmirGLO dual-luciferase vector. The wild-type, containing binding sited with the Bcl-x13'UTR or mutant, containing binding sites with deletion of the Bcl-xl were cloned downstream of firefly luciferase using the pmirGLO vector and were co-transfected with miR-133a or miR-326 in the HEK293 cells. As demonstrated in Fig. 2, in the cells transfected with the vector containing the Bcl-xl 3'UTR fragment with the binding site for Bcl-xl, the luciferase activity was significantly inhibited following transfection of miR-133a compared with the cells that were transfected with the NC miR mimics and untreated cells. Similar results were observed in the cells transfected with miR-326.

Expression levels of $\mathrm{miR}-133$ a and $\mathrm{miR}-326$ are significantly upregulated subsequent to transfection. Using RT-qPCR, the differences in the expression levels of miR-133a and miR-326 between the mimic-transfected cells and untreated cells were assessed. The miR expression levels in the HepG2 cells transfected with the miR-133a or miR-326 mimics were significantly upregulated compared with the control cells and the untreated cells (Fig. 3).

miR-133a and miR-326 downregulate the mRNA expression of $B c l-x l$. RT-qPCR was performed to assess whether miR-133a and miR-326 affected the mRNA expression of Bcl-xl. The data demonstrated that transfection with either miR-133a or miR-326 reduced the mRNA expression levels of $\mathrm{Bcl}-\mathrm{xl}$ compared with the negative control group, whereas the expression levels of Bax were increased, as expected (Fig. 4). This suggested that mRNA degradation may be an important mechanism underlying the miR-133a and miR-326-mediated post-transcriptional regulation of Bcl-xl.

miR-133a and miR-326 significantly alter the protein expression of $\mathrm{Bcl}-\mathrm{xl}$. In order to examine the effect of miR-133a and miR-326 on the endogenous expression of Bcl-xl, the miR-133a, miR-326 or negative control mimics were transfected into HepG 2 cells. The results demonstrated that enhanced expression of miR-133a or miR-326 significantly reduced the protein expression levels of Bcl-xl compared with negative control and mock-transfected cells, whereas the protein levels of Bax exhibited the opposite effects (Fig. 5), consistent with the changes in mRNA levels.

miR-133a and miR-326 sensitize HepG2 cells to 5-FU and $D D P$. To investigate whether miR-133a and miR-326 sensitize HepG2 cells to 5-FU and DDP, the miR-133a mimic, miR-326 mimic or negative control plasmids were transfected into the HepG2 cells. An MTT cell proliferation assay was performed to determine the rate of cell survival. The results indicated that transfection with the miR-133a or miR-326 mimics significantly reduced cell viability compared with negative control cells and the mock-transfected cells (Fig. 6).

\section{Discussion}

Thousands of human genes have been implicated as potential miR targets (26), and previous studies have suggested that the downregulation of miRs is involved in cancer progression $(27,28)$. miRs have also been reported to function as oncogenes or tumor suppressors by targeting specific cancer-associated genes $(29,30)$. Although the involvement of miRs has been observed in various types of cancer, the molecular mechanisms by which they modulate cancer progression and the behavior of cancer cells, including cell apoptosis and drug resistance, remain to be fully elucidated. In the present study, miR-133a and miR-326 sensitized the HepG2 HCC cell line to 5-FU and DDP. Furthermore, transfection of the cells with miR-133a and miR-326 led to the marked induction of HepG2 cell apoptosis. In the present study, Bcl-xl was further characterized as the common functional target of miR-133a and miR-326, using computational prediction programs, and the specific binding associations were confirmed using a dual-luciferase reporter assay. Transfection of the HepG2 cells with the miR-133a and miR-326 mimics significantly repressed the mRNA and protein expression levels of Bcl-xl in the HepG2 cells. These findings, together with those of the previous studies, indicate the importance of miR-133a and miR-326 in HepG2 drug resistance and implicate the potential application of miR-133a and miR-326 in cancer therapy.

Apoptosis is a process, which requires circumvention for tumor progression to occur. The human body removes unused or harmful cells to protect itself, however, cancer cells evolve to resist cell death, avoiding the surveillance system and surviving in the tumor environment (31). A previous study demonstrated that miR-133a is downregulated in osteosarcoma cell lines, and is able to reduce cell proliferation and promote cell apoptosis by targeting and repressing the expression of Bcl-xl and Mcl-1 (32). It has also been reported that the overexpression of miR-326 causes cell cycle arrest at the $G_{1}$ phase, delays cell proliferation and enhances apoptosis by targeting the nin one binding protein in human glioma cell lines (33). An additional report demonstrated that miR-326 is involved in chemotherapy resistance in breast cancer by directly modulating the expression of multidrug resistance-associated protein 1 (34). In the present study, the HepG2 HCC cell line was used as a novel model to investigate the apoptosis-associated genes by which miR-133a and miR-326 exert their functions. Bcl-xl was confirmed as a common target of miR-133a and miR-326, and miR-133a and miR-326 were confirmed as being important in the promotion of apoptosis and cancer cell drug resistance. The findings of the present study, and the previously mentioned studies, demonstrated that miR-133a and miR-326 may target multiple proteins, which function in or cooperate in different cellular processes, including cell apoptosis.

In conclusion, the present study demonstrated the functions of miR-133a and miR-326 in the regulation of $\mathrm{Bcl}-\mathrm{xl}$ expression, and in Bcl-xl-mediated cell apoptosis and drug resistance. These results contribute to a further understanding of apoptosis-associated multiple drug resistance in tumor cells. Additionally, as the introduction of a single miR can modulate complex genes, miRs may be used as potential prognostic markers and therapeutic targets for the treatment of HCC. 


\section{Acknowledgements}

This study was supported by grants from the National Natural Science Foundation of China (grant nos. 81372579 and 30900625); the Science and Technology Department Projects of Hunan Province (grant no. 2012FJ2016); the Science and Technology Development Project of Hengyang (grant no. 2012KS13); the Hunan and Innovation Fund Project of the Hunan Higher Education Institution (grant no. 13K084) and the Construction Projects of Provincial Key Disciplines.

\section{References}

1. Witko-Sarsat V: Apoptosis, cell death and inflammation. J Innate Immun 2: 201-203, 2010.

2. Ghatage DD, Gosavi SR, Ganvir SM and Hazarey VK: Apoptosis: Molecular mechanism. J Orofac Sci 4: 103-107, 2012.

3. Schultz DR and Harrington WJ Jr: Apoptosis: Programmed cell death at a molecular level. Semin Arthritis Rheum 32: 345-369, 2003.

4. Zinkel S, Gross A and Yang E: BCL2 family in DNA damage and cell cycle control. Cell Death Differ 13: 1351-1359, 2006.

5. Danial NN and Korsmeyer SJ: Cell death: critical control points. Cell 116: 205-219, 2004.

6. Petros AM, Olejniczak ET and Fesik SW: Structural biology of the Bcl-2 family of proteins. Biochim Biophys Acta 1644: 83-94, 2004.

7. Lynam-Lennon N, Maher SG and Reynolds JV: The roles of microRNA in cancer and apoptosis. Biol Rev Camb Philos Soc 84: 55-71, 2009

8. Chen G, Umelo IA, Lv S, et al: miR-146a inhibits cell growth, cell migration and induces apoptosis in non-small cell lung cancer cells. PLoS One 8: e60317, 2013.

9. Di Leva G, Garofalo M and Croce CM: MicroRNAs in Cancer. Annu Rev Pathol 9: 287-314, 2014.

10. Bartel DP: MicroRNAs: genomics, biogenesis, mechanism and function. Cell 116: 281-297, 2004

11. Farazi TA, Hoell JI, Morozov P and Tuschl T: MicroRNAs in human cancer. Adv Exp Med Biol 774: 1-20, 2013

12. Xu J, Zhu X, Wu L, et al: MicroRNA-122 suppresses cell proliferation and induces cell apoptosis in hepatocellular carcinoma by directly targeting Wnt/ $\beta$-catenin pathway. Liver Int 32: 752-760, 2012.

13. Huang X, Huang F, Yang D, et al: Expression of microRNA-122 contributes to apoptosis in H9C2 myocytes. J Cell Mol Med 16: 2637-2646, 2012.

14. Tang Y,Zheng J, Sun Y, et al: MicroRNA-1 regulates cardiomyocyte apoptosis by targeting Bcl-2. Int Heart J 50: 377-387, 2009.

15. Xiong Y, Fang JH, Yun JP, et al: Effects of microRNA-29 on apoptosis, tumorigenicity and prognosis of hepatocellular carcinoma. Hepatology 51: 836-845, 2010.

16. Zhao A, Zeng Q, Xie X, et al: MicroRNA-125b induces cancer cell apoptosis through suppression of Bcl-2 expression. J Genet Genomics 39: 29-35, 2012.
17. Jia HY, Wang YX, Yan WT, et al: MicroRNA-125b Functions as a Tumor Suppressor in Hepatocellular Carcinoma Cells. Int J Mol Sci 13: 8762-8774, 2012.

18. Lin CJ, Gong HY, Tseng HC, et al: miR-122 targets an anti-apoptotic gene, Bcl-w, in human hepatocellular carcinoma cell lines. Biochem Biophys Res Commun 375: 315-320, 2008.

19. Kontos CK, Christodoulou MI and Scorilas A: Apoptosis-related BCL2-Family members: Key players in chemotherapy. Anticancer Agents Med Chem 14: 353-374, 2014.

20. Qiu T, Zhou L, Wang T, et al: miR-503 regulates the resistance of non-small cell lung cancer cells to cisplatin by targeting Bcl-2. Int J Mol Med 32: 593-598, 2013.

21. Yang X, Yin J, Yu J, et al: miRNA-195 sensitizes human hepatocellular carcinoma cells to 5-FU by targeting BCL-w. Oncol Rep 27: 250-257, 2012.

22. Qin B, Xiao B, Liang D, et al: MicroRNA let-7c inhibits Bcl-xl expression and regulates ox-LDL-induced endothelial apoptosis. BMB Rep 45: 464-469, 2012.

23. Guo R, Wang Y, Shi WY, et al: MicroRNA miR-491-5p targeting both TP53 and Bcl-XL induces cell apoptosis in SW1990 pancreatic cancer cells through mitochondria-mediated pathway. Molecules 17: 14733-14747, 2012.

24. Ling HY, Ou HS, Feng SD, et al: CHANGES IN microRNA $(\mathrm{miR})$ profile and effects of miR-320 in insulin-resistant 3T3-L1 adipocytes. Clin Exp Pharmacol Physiol 36: e32-39, 2009.

25. Livak KJ and Schmittgen TD: Analysis of relative gene expression data using real-time quantitative PCR and the $2\left({ }^{-\Delta \Delta} C_{T}\right)$ Method. Methods 25: 402-408, 2001.

26. Lewis BP, Burge CB and Bartel DP: Conserved seed pairing, often flanked by adenosines, indicates that thousands of human genes are microRNA targets. Cell 120: 15-20, 2005.

27. Khatri R and Subramanian S: MicroRNA-135b and Its circuitry networks as potential therapeutic targets in colon cancer. Front Oncol 3: 268, 2013.

28. Nian W, Ao X, Wu Y, et al: miR-223 functions as a potent tumor suppressor of the Lewis lung carcinoma cell line by targeting insulin-like growth factor-1 receptor and cyclin-dependent kinase 2. Oncol Lett 6: 359-366, 2013.

29. Georgantas RW, Streicher K, Zhu W, et al: MicroRNA oncogenes and tumor suppressors controlling malignant melanoma cell growth, apoptosis, migration, and invasion. J Clin Oncol 29: $8549,2011$.

30. Wang XF, Shi ZM, Wang XR, et al: MiR-181d acts as a tumor suppressor in glioma by targeting K-ras and Bcl-2. J Cancer Res Clin Oncol 138: 573-584, 2012.

31. Hanahan D and Weinberg RA: Hallmarks of cancer: the next generation. Cell 144: 646-674, 2011.

32. Ji F, Zhang H, Wang Y, et al: MicroRNA-133a, downregulated in osteosarcoma, suppresses proliferation and promotes apoptosis by targeting Bcl-xl and Mcl-1. Bone 56: 220-226, 2013.

33. Zhou J, Xu T, Yan Y, et al: MicroRNA-326 functions as a tumor suppressor in glioma by targeting the Nin one binding protein (NOB1). PLoS One 8: e68469, 2013.

34. Liang Z, Wu H, Xia J, et al: Involvement of miR-326 in chemotherapy resistance of breast cancer through modulating expression of multidrug resistance-associated protein 1 . Biochem Pharmacol 79: 817-824, 2010. 